\title{
Divertículo congênito do ventrículo esquerdo associado a defeitos da parede tóraco-abdominal: relato de paciente operada em idade adulta
}

Roney Ronald Peixoto da SILVA*, Homero Geraldo de OLIVEIRA*, Renato Rocha RABELLO*, Sérgio Caporali de OLIVEIRA*, Sebastiāo Correa RABELLO*

\begin{abstract}
SILVA, R. R. P.; OLIVEIRA, H. G.; RABELLO, R. R.; OLIVEIRA, S. C.; RABELLO, S. C. - Divertículo congênito do ventrículo esquerdo associado a defeitos da parede tóraco-abdominal: relato de paciente operada em idade adulta. Rev. Bras. Cir. Cardiovasc. 7(1):51-55, 1992.
\end{abstract}

RESUMO: O divertículo congênito do ventrículo esquerdo é anomalia rara. Pode estar associado a defeitos da parede tóraco-abdominal e a lesōes intracardíacas. Quando isto ocorre, recebe o nome de síndrome de Cantrell. É relatado o caso de paciente de 25 anos de idade submetida a tratamento cirúrgico.

DESCRITORES: ventrículo esquerdo, divertículo, cirurgia; síndrome de Cantrell.

\section{INTRODUÇÃO}

A deformidade sacular do ventrículo cardíaco pode ser um aneurisma, uma hérnia através de defeito parcial do pericárdio, ou um divertículo.

O divertículo cardíaco, malformação congênita, apresenta as três camadas normais da parede do coração (endocárdio, miocárdio e epicárdio), e tem atividade mecânica sincrônica com a atividade ventricular.

Normalmente é do ventrículo esquerdo $1,3-7,10-13,15$. 20, 22, 23, mas existem casos relatados de divertículos de ventrículo direito ${ }^{8,9,14}$ e até de ambos ${ }^{2}$.

O primeiro caso foi descrito por O'Brian, em 1838 , e diversos autores ${ }^{1-23}$ destacam a pequena incidência na literatura.

CANTRELL et alii ${ }^{4}$ observaram uma significativa freqüência da associação de divertículos ventriculares comoutras anomalias, em uma série de pacientes. As anomalias observadas eram: 1 ) defeito da parede abdominal, supra-umbilical mediana; 2) defeito da porção inferior do esterno; 3) defeito da porção anterior do diafragma; 4) defeito no pericárdio diafragmático; 5) lesōes congênitas intrcardíacas. Propuseram uma síndrome específica, uma provável base embriológica e consideraram o tratamento cirúrgico.

A quase totalidade dos relatos da literatura são de operações realizadas em crianças de baixa idade e recém-nascidos.

Os autores relatam o caso de uma paciente adulta, portadora de divertículo de ventrículo esquerdo, associado a defeitos da parede tóracoabdominal, diafragma e pericárdio, operada com sucesso.

\section{RELATO DO CASO}

V.A.S., sexo feminino, 25 anos de idade, branca, nascida de parto domiciliar a termo, sem complicaçōes, com índice gestacional adequado.

Aos 4 meses de idade, a família foi informada

Trabalho realizado no Hospital Vera Cruz. Belo Horizonte, MG, Brasil.

* Do Hospital Vera Cruz.

Recebido para publicação em 28 de março de 1992.

Endereço para separatas: Roney Ronald Peixoto da Silva. Av. Barbacena, 653 - Barro Preto. 30190 Belo Horizonte, MG, Brasil. 
SILVA, R. R. P.; OLIVEIRA, H. G.; RABELLO, R. R.; OLIVEIRA, S. C.; RABELLO, S. C. - Divertículo congênito do ventrículo esquerdo associado a defeitos da parede tóraco-abdominal: relato de paciente operada em idade adulta. Rev. Bras. Cir. Cardiovasc. $7(1): 51-55,1992$.

pelo pediatra da presença de uma deformidade cardíaca congênita, tendo sido alertada para a possibilidade de correção cirúrgica, que foi rejeitada.

Evoluiu assintomática, sem controle médico até aos 25 anos, quando começou a sentir desconforto apigástrico, provocado por palpitaçōes.

Encaminhada ao nosso Serviço, apresentava, ao exame clínico, bom estado geral, com ausência do terço inferior do esterno, diástase da musculatura reto-abdominal e hérnia epigástrica, cujo conteúdo pulsava sincronicamente com os batimentos cardíacos.

O ritmo cardíaco era regular em dois tempos, ausência de sopros, B3 ou B4. A radiografia do tórax revelava discreto aumento da área cardíaca, arco médio proeminente e circulação pulmonar espessada. O ECG mostrava RSR com inversão de onda Tem D II, D III e aVF.

Foi submetida a estudo hemodinâmico, que constatou pressões normais em cavidades cardíacas, ausência de shunts, presença de divertículo muscular do ventrículo esquerdo e miocardiopatia do ventrículo direito (Figura 1).

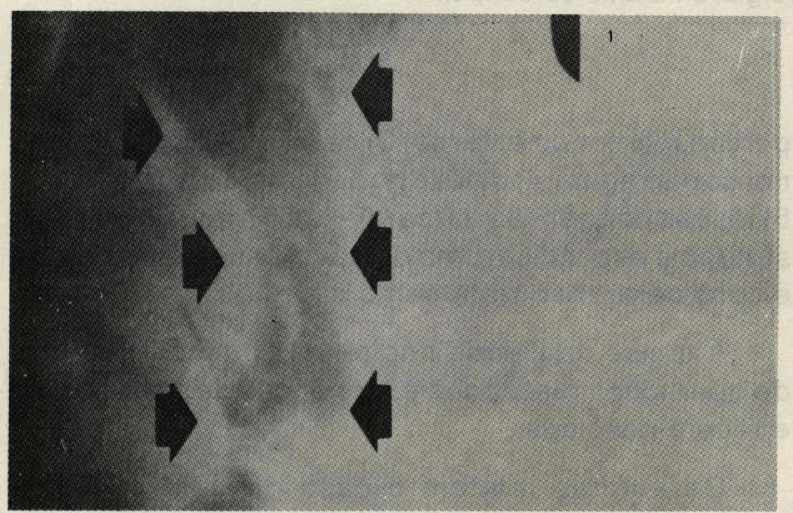

Fig. 1 - Imagem contrastada do divertículo durante ventriculografia esquerda, em diástole, em oblíqua anterior esquerda.

A paciente foi submetida a cirirgia através de esternotomia mediana; identificado divertículo de ventrículo esquerdo, com cerca de $6 \mathrm{~cm}$ de comprometimento, com início no ápice junto ao término da artéria interventricular anterior. Migrava para o abdome através de orifício do diafragma de cerda de $4 \mathrm{~cm}$ de diâmetro. Era destituído de pericárdio parietal. O coração apresentava-se rodado para a direita, estando o ápice na linha média.

Notava-se, ainda, ausência do processo xifóide e fusão incompleta do têrço distal do esterno.

Instalou-se a circulação extracorpórea (CEC) de maneira usual, em normotermia. Não se realizou

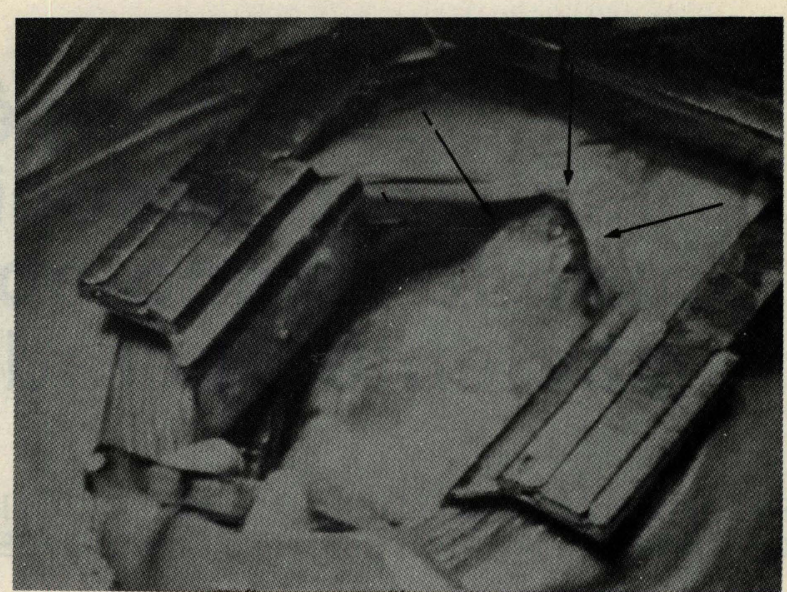

Fig. 2 - Esternotomia mediana, expondo o coração. As setas apontam a migração do divertículo esquerdo para o abdome através de defeito do diafragma.

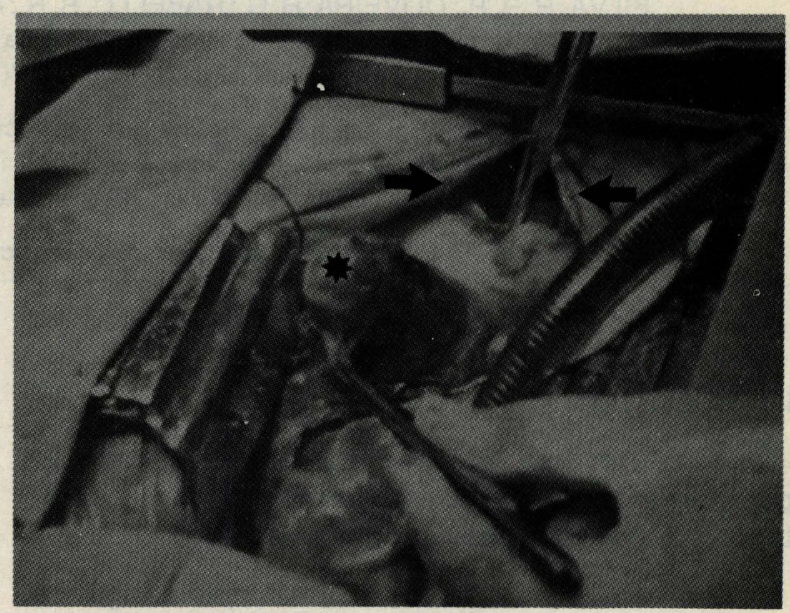

Fig. 3-O asterisco identifica o divertículo do ventrículo esquerdo luxado para expor o defeito da parede diafragmática mostrado pelas setas.

pinçamento da aorta. Ressecou-se o divertículo, junto da zona normal da parede do ventrículo esquerdo.

Não havia trombos intracavitários e a implantação dos músculos papilares da valva mitral não estavamenvolvidos na lesão. Procedeu-se a ventriculorrafia por técnica usual.

Após a descontinuação da CEC, prolongou-se a incisão em seu ângulo inferior até a cicatriz umbilical.

Ocluiu-se o orifício herniário diafragmático por meio de técnica clássica. Procedeu-se a reconstituição da parede abdominal, a esternorrafia e a sutura da pele.

A paciente evoluiu sem intercorrência e, no $13^{\circ}$ dia de pós-operatório, recebeu alta hospitalar em boas condições. 
SILVA, R. R. P.; OLIVEIRA, H. G.; RABELLO, R. R.; OLIVEIRA, S. C.; RABELLO, S. C. - Divertículo congênito do ventrículo esquerdo associado a defeitos da parede tóraco-abdominal: relato de paciente operada em idade adulta. Rev. Bras. Cir. Cardiovasc. $7(1): 51-55,1992$.

Já no seu domicílio, observou epigastralgia que aparecia após a alimentação e cedia com vômitos, motivo pelo qual retornou à consulta, sendo reinternada no $60^{\circ}$ dia de pós-operatório, para investigação e tratamento.

Foi submetida a exame radiológico contrastado do trato gastrintestinal superior, que não evidenciou alteraçōes dignas de nota. Obteve melhora espontânea e no $8^{\circ}$ dia dessa segunda internação, recebeu alta hospitalar.

Atualmente, está sendo acompanhada em nosso ambulatório, encontrando-se assintomática há 50 meses da cirurgia. Nesse intervalo, casou-se e teve uma gravidez levada a termo, sem complicações.

O exame anatomopatológico do divertículo mostrou a presença das três camadas cardíacas, com hipertrofia moderada de fibrocélulas miocárdicas, miocardioesclerose moderada sem sinais inflamatórios ou degenerativos (Figura 4).

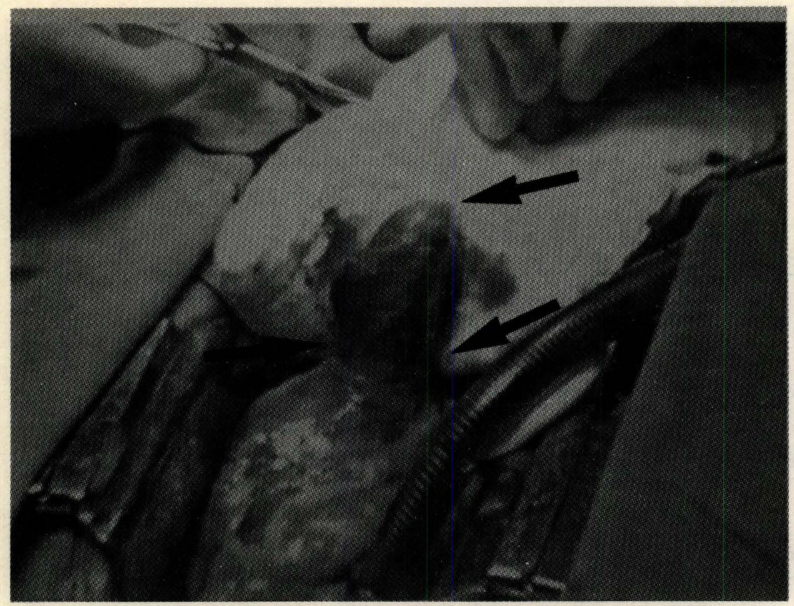

Fig. 4 - As setas apontam para o divertículo do ventrículo esquerdo. que media $6 \mathrm{~cm}$ de comprimento.

\section{COMENTÁRIOS}

O divertículo congênito do ventrículo esquerdo é anomalia muito rara. Foi descrito pela primeira vez, em 1838, por O'Bryan.

Sua incidência foi estimada em $0,013 \%$ por Keith, segundo HALLALI et alli ${ }^{\circ}$, e de $0,076 \%$ em mais de 13000 casos operados por cardiopatia congênita no Texas Heart Institute, Houston ${ }^{17}$.

Em 1958, CANTRELL et alii ${ }^{4}$ propuseram uma síndrome específica, na qual o divertículo ventricular estava associado a defeitos congênitos da parede abdominal, do esterno, do diafragma, do pericárdio e lesōes intracardíacas. Propuseram uma base embriológica e fizeram comentários sobre o tratamento cirúrgico.
TOYAMA ${ }^{21}$, em 1972, fez o relato de um caso de criança nascida com defeitos na parede tóraco-abdominal, com grande hérnia epigástrica e diversas lesões intracardíacas identificadas na necropsia.

Fez a revisão de todos os casos publicados e qüestionou os exatos critérios da síndrome proposta por Cantrell. Subdividiu os casos em três grandes grupos: o primeiro incluía aqueles casos em que os cinco defeitos estavam bem diagnosticados e relatados; o segundo grupo incluía os casos em que existia alguma dúvida, se todas as cinco lesōes estavam bem definidas, ou se havia descrição incompleta dos defeitos; no terceiro grupo, incluiu os casos em que, sem dúvida, havia expressão incompleta da síndrome.

BALTAXE et alii ${ }^{3}$ reconhecem três grandes grupos de divertículos ventriculares na literatura. O primeiro corresponde à síndrome proposta por Cantrell, porém, ressaltam a grande variedade de lesōes intracardíacas. No segundo grupo, o divertículo ventricular é subvalvar e está intimamente relacionado às valvas mitral e aórtica. Essa lesão ocorre em nigerianos, índios Bantus e negros americanos. O terceiro grupo é constituído por pacientes portadores de divertículo do ápice do ventrículo esquerdo, sem associação com defeitos da parede tóraco-abdominal. Nesse trabalho, os autores relatam o achado de nove pacientes portadores de divertículos ventriculares em posiçōes diversas da ápice. Esses pacientes não apresentavam outras anomalias e não tinham correlação racial.

MILLER \& MATTEWS ${ }^{14}$ relataram o caso de uma criança com divertículo ventricular, associado a outras lesões cardíacas e defeitos da parede tóracoabdominal e alertaram que, em virtude do desenvolvimento embriológico dos defeitos, variações na síndrome proposta por Cantrell são esperadas.

SIMBAS \& WARE ${ }^{20}$ relataram o caso de uma criança de 6 anos, portadora de divertículo de ventrículo esquerdo, associado a defeitos da parede abdominal, sem lesōes intracardíacas, operada com utilização de circulação extracorpórea. Citam a revisão bibliográfica de MURPHY et alli ${ }^{16}$, na qual $22 \%$ dos casos relatados não apresentam defeitos intracardíacos e propõem a simplificação dos critérios para inclusão na síndrome proposta por Cantrell, conforme o seguinte: 1) defeito da linha média da parede abdominal superior; 2) defeito da porção inferior do esterno; 3) defeito do diafragma ventral e pericárdio adjacente; 4) anomalias cardíacas, que poderiam variar desde uma simples destro-rotação, até lesōes mais complexas, com a tetralogia de Fallot.

Como o caso por nós relatado se enquadra nos critérios acima discutidos, juigamos procedente a sua inclusão na síndrome proposta por Cantrell.

Deve-se considerar, também, que é extrema- 
SILVA, R. R. P.; OLIVEIRA, H. G.; RABELLO, R. R.; OLIVEIRA, S. C.; RABELLO, S. C. - Divertículo congênito do ventrículo esquerdo associado a defeitos da parede tóraco-abdominal: relato de paciente operada em idade adulta. Rev. Bras. Cir. Cardiovasc. $7(1): 51-55,1992$.

mente raro o achado em pacientes na fase adulta. MADY ${ }^{12}$ relata apenas quatro casos de pacientes acima de 20 anos, em sua revisão bibliográfica, e SUILEN et alii ${ }^{19}$ relatam seis casos.

São descritas, como causa mortis, a rotura espontânea do divertículo, as taquiarritmias, a insuficiência cardíaca e as embolias.

Devemos levar em consideração que a presença de defeitos intracardíacos é fundamental na sobrevida do paciente e que a sua ausência no caso por nós relatado seguramente permitiu à paciente atingir a fase adulta.

A indicação cirúrgica é absoluta e deve, sempre que possível, levar em consideração a abordagem simultânea dos defeitos cardíacos.

MADY ${ }^{12}$, CHRISTO et alii ${ }^{5}$ e OSSANDON ${ }^{18}$ relatam a ressecção do divertículo sem CEC. Porém EDGETT Jr. et alii ${ }^{7}$, CANTRELL et alii ${ }^{4}$ e AKKARY et alii ${ }^{1}$ relatam óbitos per-operatórios que podem estar relacionados diretamente à ressecção do divertículo sem auxílio da CEC.

SUILEN et alii ${ }^{19}$ relatam o caso de uma paciente de 53 anos de idade submetida a ressecção de um divertículo isolado, com utilização de CEC.

OKEREKE et alii ${ }^{17}$ fizeram a ressecção do divertículo ventricular em três casos sem CEC. Em seis utilizaram a CEC: em três, pela presença de lesões intracavitárias e, em outros três, por não ter sido possível pinçar o divertículo.

SYMBAS \& WARE ${ }^{20}$ mostram que o pinçamento do divertículo pode envolver seriamente a base do músculo papilar anterior da valva mitral, considerando a ressecção com auxílio da CEC a forma mais segura para o paciente.

HÉLARDOT et alii ${ }^{10} \mathrm{e}$ GILLE et alii ${ }^{8}$ mostram que é possível o diagnóstico pré-natal do divertículo através da ultrassonografia, o que possibilitaria a abordagem dos pacientes no período neonatal.

\section{RBCCV 44205-163}

SILVA, R. R. P.; OLIVEIRA, H. G.; RABELLO, R. R.; OLIVEIRA, S. C.; RABELLO, S. C. - Muscular diverticulum of the left ventricle associated with anomalies of the abdominal wall: report of an adult patient operated on. Rev. Bras. Cir. Cardiovasc., 7(1):51-55, 1992.

ABSTRACT: Muscular diverticulum of the left ventricle is a rare anomaly. It is frequently associated with other congenital anomalies of the abdominal wall, sternum, diaphragm, pericardium and the heart itself. These have been described as Cantrell syndrome. This paper reports a case of a 25 -year-old female patient submitted to surgical repair of this syndrome.

DESCRIPTORS: left ventricle, diverticulum, surgery; Cantrell syndrome.

\section{REFERÊNCIAS BIBLIOGRÁFICAS}

1 AKKARY, S.; SAHWI, E.; KANDIL, W. - Congenital diverticulum of the left ventricle. J. Pediatr. Surg., 16 737-738, 1981.

ASHRAF, M. H.; GINGELL, R.; PIERONI, D.; DHAR, N.; SUBRAMANIAN, S. - Congenital diverticulum of the heart of biventricular origin. Thorac. Cardiovasc. Surg., 32: 389-391, 1984.

BALTAXE, H. A.; WILSON, W. J.; AMIEL, M. - Diverticulosis of the left ventricle. Am J. Roentgenol., 133: 257$261,1979$. syndrome of congenital defects involving the abdo-

minal wall, sternum, diaphragm, pericardium, and heart. Surg. Gynecol. Obstet., 107: 602-614, 1958.

5

CHRISTO, M, C.; SOUZA, J. M.; STORTINI, M. J.; FIGUEROA, C. S.; SANTANA FILHO, G. P.; GOMES, M. V. - Divertículo congênito do ventrículo esquerdo: relato de um caso operado. Arq. Bras. Cardiol., 29:241-244, 1976.

6 EBAID, M.; GREGORI JÚNIOR, F.; BITTENCOURT, D.; FUJIOKA, T.; MACRUZ, R.; MOFFA, P. J.; DÉCOURT, L. V. - Divertículo congênito de ventrículo esquerdo: aspectos clínicose hemodinâmicos. Arq. Bras. Cardiol., 28: 213-219, 1975.

7 EDGETT Jr., J. W.; NELSON, W. P.; HALL, R. J.; FISHBACK, M. E.; JAHNKE, E. J.; - Diverticulum of 
SILVA, R. R. P.; OLIVEIRA, H. G.; RABELLO, R. R.; OLIVEIRA, S. C.; RABELLO, S. C. - Divertículo congênito do ventrículo esquerdo associado a defeitos da parede tóraco-abdominal: relato de paciente operada em idade adulta. Rev. Bras. Cir. Cardiovasc. $7(1): 51-55,1992$.

the heart. Am. J. Cardiol., 24: 580-583, 1969.

8

GILLE, P.; VERNEY,R.; MELEY, M.;AUBERT,D.;DELAFIN, J.; MOUROT, M. - Diverticule congénital épigastrique du ventricule droit. Chir. Pédiatr., 20:349-354, 1979.

9 HALLALI, P.; IUNG, B.; DAVIDO, A.; BINET, J.; LERICHE, $H . ;$ CORONE, P. - Congenital diverticulum of the right ventricle: report of two cases associated with other congenital heart defects. Am. Heart J., 117: 957959, 1989.

10 HANDLER, C. E. \& WALKER, J. M. - Congenital diverticulum of the left ventricle presenting as heart failure and diagnosed by magnetic resonance imaging. Internat. J. Cardiol., 22: 115-119, 1989.

11 HÉLARDOT,P. G.; BARGY,F.; BIENAIMÉ, J. - Le diagnostic antenatal des malformations viscélares par ultrasonographie. Chir. Pédiatr., 24: 105-108, 1983.

12 MADY, C. - Left ventricular diverticulum: analysis of two operated cases and review of the literature. Angiology, 33: 280-286, 1982.

13 MARDINI, M. K. - Congenital diverticulum of the left ventricle: report of two unusual cases. Br. Heart J., 51:321-326, 1984

MILLER, J. D. \& MATTEWS, E. C. - Congenital cardiac diverticulum. Am. J. Dis. Child, 126: 814-816, 1973.

15 MULDER, D. G.; CRITTENDEN, I. H.; ADAMS, F. H. Complete repair of a syndrome of congenital defects involving the abdominal wall, sternum, diaphragm, pericardium, and heart: excision of left ventricular diverticulum. Ann. Surg.; 151: 113-122, 1960.
MURPHY, D. A.; ABERDEEN, E.; DOBBS, R. H.; WATERSTON, D. J. - The surgical treatment of a syndrome consisting of thoracoabdominal wall; diaphragmatic, pericardial, and ventricular septal defects, and a left ventricular diverticulum. Ann. Thorac. Surg., 6: 528-534, 1968.

OKEREKE, O. U. J.; COOLEY, D. A.; FRAZIER, O. H. Congenital diverticulum of the ventricle. J. Thorac. Cardiovasc. Surg., 91: 208-214, 1986.

OSSANDON, F. - Congenital diverticulum of the left ventricle extending into exomphalos. $Z$. Kinderchir. - Grenzgeb., 30:265-266, 1980.

SUILEN, C.; FRIEDLI, B.; RUTISHAUSER, W. - Congenital intrathoracic left ventricular diverticulum in an adult. Chest, 98: 750-751, 1990.

SYMBAS, P. N. \& WARE, R. E. - A syndrome of defects of the thoracoabdominal wall, diaphragm, pericardium, and heart. J. Thorac. Cardiovasc. Surg., 65: 914$919,1973$.

TOYAMA, W. M. - Combined congenital defects of the anterior abdominal wall, sternum, diaphragm, pericardium, and heart: a case report and review of the syndrome. Pediatrics, 50: 778-792, 1972.

VANCHERI, F.; TROVATO, G. M.; SHINEBOURNE, E. A. - Isolated congenital left ventricular diverticulum. Int. J. Cardiol., 22: 122-126, 1989.

VERGER, H. B.; FONTAN, F.; GUILLARD, J. M.; MOUNICOT, F. B.; SANDLER, B.; SAGARDILUZ, J. - Le diverticule congénital du ventricule gauche. Ann. Pédiatr., 17:24-30, 1970. 